\title{
BMJ Open Impact of carbapenem resistance on mortality in patients infected with Enterobacteriaceae: a systematic review and meta-analysis
}

Ruyin Zhou (D) , Xiangming Fang, ${ }^{1,2}$ Jinjin Zhang, ${ }^{1}$ Xiaodong Zheng, ${ }^{3}$
Shuangyue Shangguan, ${ }^{1}$ Shibo Chen, ${ }^{4}$ Yingbo Shen, ${ }^{5}$ Zhihai Liu, ${ }^{6}$ Juan Li, ${ }^{7}$
Rong Zhang, ${ }^{8}$ Jianzhong Shen, ${ }^{9}$ Timothy R Walsh,,${ }^{10}$ Yang Wang ${ }^{9}$

To cite: Zhou R, Fang $X$, Zhang J, et al. Impact of carbapenem resistance on mortality in patients infected with Enterobacteriaceae: a systematic review and meta-analysis. BMJ Open 2021;11:e054971. doi:10.1136/ bmjopen-2021-054971

- Prepublication history and additional supplemental material for this paper are available online. To view these files, please visit the journal online (http://dx.doi.org/10.1136/ bmjopen-2021-054971)

Received 29 June 2021 Accepted 22 November 2021

Check for updates

(C) Author(s) (or their employer(s)) 2021. Re-use permitted under CC BY-NC. No commercial re-use. See rights and permissions. Published by BMJ.

For numbered affiliations see end of article.

Correspondence to

Xiangming Fang;

xmfang@cau.edu.cn

\section{ABSTRACT}

Objectives To provide a comprehensive assessment of the impact of carbapenem resistance on mortality among patients infected with Enterobacteriaceae and to explore the source of heterogeneity across studies.

Design This systematic review was conducted following the guidelines of Cochrane Guidance and the Preferred Reporting Items for Systematic Reviews and Meta-Analyses.

Data sources We conducted a systematic literature search of the PubMed, Embase, Web of Science and Cochrane Library databases to identify relevant studies published between 1 January 1994 and 30 August 2020. Eligibility criteria We included primary observational studies published in English that reported the mortality outcomes for hospitalised patients with confirmed infections due to carbapenem-resistant Enterobacteriaceae (CRE) and carbapenem-susceptible Enterobacteriaceae (CSE). Studies with no comparison group or with a comparison group of patients infected with unconfirmed CSE were excluded. Data extraction and synthesis Data extraction and assessment of risk bias were conducted independently by two reviewers. The pooled relative risk and risk difference were calculated as effect measures with $95 \%$ Cls using a random effects model. The heterogeneity across studies was assessed by $Q$-statistic and $I^{2}$ measures.

Results Of 10304 studies initially identified, 50 studies were included in the meta-analyses. The results of the meta-analyses showed that carbapenem resistance has a significant positive effect on the probability of death for patients infected with Enterobacteriaceae for any type of mortality outcome. The results of the stratified analysis and meta-regression suggested that the effect of carbapenem resistance on the risk of death varied by infection type, sample size and year of publication.

Conclusions Our results suggested that patients with CRE infection still face a greater risk of death than patients with CSE infection do, and an urgent need to develop new antibiotics and appropriate treatments to reduce the risk of death.

PROSPERO registration number CRD42020176808.

\section{INTRODUCTION}

The Enterobacteriaceae species, mainly Klebsiella pneumoniae and Escherichia coli, can cause infections like bloodstream infections,

\section{Strengths and limitations of this study}

- This study provided a comprehensive metaanalysis to assess the impact of carbapenem resistance on mortality among patients infected with Enterobacteriaceae, including nearly 20 new published studies in the last 3 years that were not included in previous relevant reviews.

- The statistical test and meta-regression analysis in this study were conducted for different groups of mortality outcome type, which may help to address the potential heterogeneity caused by the factor of mortality measurements.

- This review is the first to explore the source of heterogeneity across studies through meta-regression analysis and to consider the country's economic status and geographical region in assessing the association between carbapenem resistance and mortality among patients infected with Enterobacteriaceae.

- This review includes effect measures in both relative and absolute terms, thus providing a complete picture of the effect of carbapenem resistance on mortality among patients infected with Enterobacteriaceae.

- The comparison in our research is currently limited to high-income and upper middle-income countries from the Americas, Asia and Europe due to insufficient data from elsewhere; more studies from different countries, especially low-income countries and other regions, are needed to provide comprehensive data for further analysis stratified by geographical region and economic status.

ventilator-associated pneumonia, intraabdominal infections and urinary tract infections in both healthcare and community settings. ${ }^{1}$ The treatment of these infections is becoming increasingly challenging because of the increasing prevalence of multidrugresistant Enterobacteriaceae, such as extendedspectrum $\beta$-lactamases (ESBLs)-producing Enterobacteriaceae. To counter this challenge, carbapenems were introduced in the $1980 \mathrm{~s}^{2}$ 
and proved efficacious in the clinical treatment of infections caused by ESBLs-producing Enterobacteriaceae. ${ }^{3}$ However, since the carbapenem-resistant Enterobacteriaceae (CRE) emerged in the early 1990s, ${ }^{4}$ CRE has been increasingly reported worldwide. ${ }^{56}$ The prevalence of CRE is driven primarily by the spread of carbapenemases, a group of $\beta$-lactamases hydrolysing carbapenems. ${ }^{7}$ The CRE strains that produce diverse types of carbapenemases are endemic in different areas of the world. ${ }^{8}$ Countries that have high overall rates of CRE include Greece, Italy, Brazil, China, the USA and Colombia. ${ }^{7}$ For example, the rate of carbapenem resistance in Klebsiella pneumoniae isolates was as high as $63.9 \%$ in Greece in $2018 .{ }^{9}$ The increasing prevalence of CRE has posed a serious threat to public health because of the reduced efficacy of carbapenem and limited available therapy options, so CRE has been categorised as the most critical group of multidrug-resistant pathogens with the most urgent need for new antibiotics. ${ }^{10}$

The mortality of CRE infections is a research hotspot. Recently, some systematic reviews have included metaanalyses to assess the association between CRE infections and mortality by comparing with the mortality outcome of patients infected with carbapenem-susceptible Enterobacteriaceae (CSE) ${ }^{11-16}$ The results showed that CRE infections could lead to increased mortality. The latest systematic review on this topic included studies published until $2017,{ }^{12}$ but nearly 20 relevant articles have been published since then. A timely and comprehensive summary of the results of these articles can help explain the excess health burden that is attributable to CRE infections. Moreover, although previous systematic reviews have identified heterogeneity across studies and discussed some confounding factors of mortality, including patient-related, infection-related, organism-related and therapy-related factors, ${ }^{12} 1315$ few used a formal statistical approach or meta-regression analysis to examine whether the effect of carbapenem resistance on mortality varies by these factors. In addition, these earlier reviews have not considered differences in economic status and geographical region. The development of antibiotic resistance has resulted in decreasing effectiveness of first-line antibiotics, such that more expensive second-line and third-line antibiotic treatments must be used. However, these treatments may be unobtainable or unaffordable for patients with resistant infections in developing countries, ${ }^{17}$ which would result in worse prognostic outcomes. The effect of carbapenem resistance on mortality may have regional differences because the CRE strains with different types of carbapenemases and virulence characteristics ${ }^{1}$ are predominant in different regions worldwide. ${ }^{8}$ Two previous reviews have shown that the mortality rate of patients with CRE infections differs by geographical region. ${ }^{14}{ }^{18}$ However, without data from patients with CSE infections, whether the impact of carbapenem resistance on mortality differs between the region will remain unknown.

Therefore, we aim to provide a comprehensive systematic review of the impact of carbapenem resistance on mortality among patients infected with Enterobacteriaceae and explore the source of heterogeneity among studies to help policymakers to develop strategies and policies to combat CRE worldwide.

\section{METHODS}

This systematic review was conducted following the guidelines of Cochrane Guidance ${ }^{19}$ and the Preferred Reporting Items for Systematic Reviews and Meta-Analyses. ${ }^{20}$ The protocol was registered with PROSPERO on 5 July 2020. The initial protocol was designed with a broad scope, but we divided our work into two parts to limit its length: the first (this study) focuses on mortality and the second will focus on morbidity and the economic outcomes.

\section{Search strategy}

We conducted a systematic literature search of the databases of PubMed, Embase, Web of Science and the Cochrane Library for relevant studies published between 1 January 1994 and 30 August 2020 to identify eligible studies. This period was chosen because CRE were first reported in the 1990s. Specifically, the strains producing metallo- $\beta$-lactamase imipenemase 1 (IMP- 1 ), which is a type of carbapenemase that can hydrolyse carbapenems, were first identified in Japan in a study published in $1994 .{ }^{4}$

The search strategy was designed by combining the terms for bacteria and carbapenem resistance (see online supplemental appendix 1). The search terms for the bacteria were 'Enterobacteriaceae', along with 'Klebsiella pneumoniae' and 'Escherichia coli', (the two most clinically important pathogens within the Enterobacteriaceae family). The search terms for carbapenem resistance were 'carbapenem-resistant', 'carbapenem resistance', 'carbapenem non-susceptible' and 'carbapenemaseproducing' because CRE can be generally divided into carbapenemase-producing CRE (CP-CRE) and non-CP-CRE. ${ }^{21}$

\section{Selection criteria}

We included studies that fulfilled all of the following criteria: (1) primary observational studies (ie, casecontrol study, cohort study), (2) studies published between 1 January 1994 and 30 August 2020, (3) studies published in English and (4) studies that assessed the mortality of hospitalised patients with confirmed infections due to CRE and CSE.

Studies that met any of the following criteria were excluded: (1) studies that could not provide the mortality data for patients with confirmed CRE infection, (2) studies that focused on the resistance of other antibiotics instead of carbapenem antibiotics, (3) studies with no comparison group or with a comparison group of patients infected with unconfirmed CSE, (4) studies on animals or (5) publications like editorials and letters. The list of excluded studies with reasons for exclusion is provided in online supplemental appendix 2. 
Two reviewers independently screened all titles and abstracts of the initially identified studies and then reviewed the full text of studies that met all of the inclusion criteria and none of the exclusion criteria. Disagreements were resolved through consensus or discussion with a third senior reviewer.

\section{Data extraction}

Data were extracted from each selected study into a data extraction form in Excel. The extracted data included the first author, year of publication, study period, country, region, country income level classified by the World Bank, ${ }^{22}$ study design, infection type, specific pathogen, sample size and the number of deaths in CRE and CSE groups. Notably, we assigned the income status of the country based on the period when the study was conducted because the income status of some countries may have changed between 1994 and 2020. For example, there were 15 studies conducted in China between 2006 and 2018 included in this meta-analysis, but since the income status of China changed from the lower income level to the upper income level in 2010, the two studies conducted between 2006 and 2009 were classified as lower middle income, and the other 13 studies conducted after 2010 were classified as upper middle income. The kinds of measurements of mortality outcomes that were extracted from included studies were all-cause in-hospital mortality, all-cause mortality at $6-30$ days ( 6 days, 7 days, 14 days, 21 days, 28 days, 30 days) after diagnosis, mortality in ICU, 30-day mortality in ICU and mortality attributable to infection, which is usually defined as the death of a patient with clinical and laboratory evidence of ongoing infection in the absence of other feasible reasons.

Data extraction was conducted by two reviewers independently and disagreements were resolved through consensus or discussion with a third senior reviewer.

\section{Data synthesis and analysis}

We calculated the pooled relative risk (RR) and risk difference (RD) by comparing the mortality of patients with CRE infection with that of patients with CSE infection. RR was chosen as the relative measure rather than the OR because the latter was more difficult to interpret than $R^{23}{ }^{24}$ and was usually misinterpreted as RR, which may overestimate the intervention effect when RR is more than $1 .{ }^{25}$ We also calculated RD to describe the absolute difference in the risk of mortality between the two groups because reporting only the RR may conceal the underlying absolute risks, resulting in readers' overestimating the effect. ${ }^{26}$ It has been recommended that both RR and absolute risk should be reported to provide a complete picture of the effect. ${ }^{27}$ We calculated the pooled estimates of RRs and RDs with 95\% CIs were calculated using a random effects model based on the method of DerSimonian and Laird, ${ }^{28}$ with the estimate of heterogeneity being taken from the Mantel-Haenszel model. An RR of 1 and an RD of 0 indicate that the risk of mortality is identical regardless of carbapenem resistance. When
$\mathrm{RR}>1$ or $\mathrm{RD}>0$, it means carbapenem resistance has a positive effect on the risk of death for patients infected with Enterobacteriaceae, in other words, the risk of death from CRE infection is higher than that from CSE infection. The heterogeneity across studies was assessed by Q-statistic and $\mathrm{I}^{2}$ measures. The heterogeneity was considered substantial when $\mathrm{I}^{2}>50 \%$.

In the primary analysis, we calculated the pooled estimates of the overall mortality using one mortality outcome in each study with a priority given to in-hospital mortality and the latest time point of mortality if mortality outcomes at multiple time points were reported in a study. Then, we categorised the mortality measurements into eight groups and conducted meta-analysis for each type of mortality outcome. In further analysis, to identify the potential sources of heterogeneity, we conducted stratified analysis by bacterial species, geographical region, economic status, source of infection, sample size and resistance mechanism in the mortality outcome groups, in which substantial heterogeneity was detected. An F test based on a one-way analysis of variance was used to test the differences in the mean effect estimates between subgroups. In the groups of mortality outcome type with more than ten studies, we also conducted the randomeffects meta-regression analyses. The meta-regression analysis was based on restricted maximum likelihood using an iterative procedure to determine whether the effect estimates differ significantly by the above variables, and $p<0.1$ was considered statistically significant. A sensitivity analysis was conducted for the overall mortality, with the pooled RRs recalculated using random effects metaanalysis after removing one study at a time to evaluate the stability of the results. Finally, we conducted a funnel plot for the overall mortality to assess the publication bias. All the statistical analyses were conducted using the Stata V.15 software.

\section{Risk of bias assessment}

Two reviewers independently assessed the risk of bias for each included study using the Newcastle-Ottawa quality assessment scale (NOS) for observational studies, ${ }^{29}$ and disagreements were resolved through consensus or discussion with a third senior reviewer.

\section{Patient and public involvement}

Patients and the public were not involved in the design, or conduct, or reporting or dissemination plans of this systematic review.

\section{RESULTS}

We identified 10304 studies from the literature search, among which 50 studies $^{30-79}$ were selected for final review based on the inclusion and exclusion criteria (figure 1). The basic characteristics of the included studies are provided in table 1 , and online supplemental table 1 shows the details of the studies. The studies were conducted in 14 countries from four regions. Nearly half 


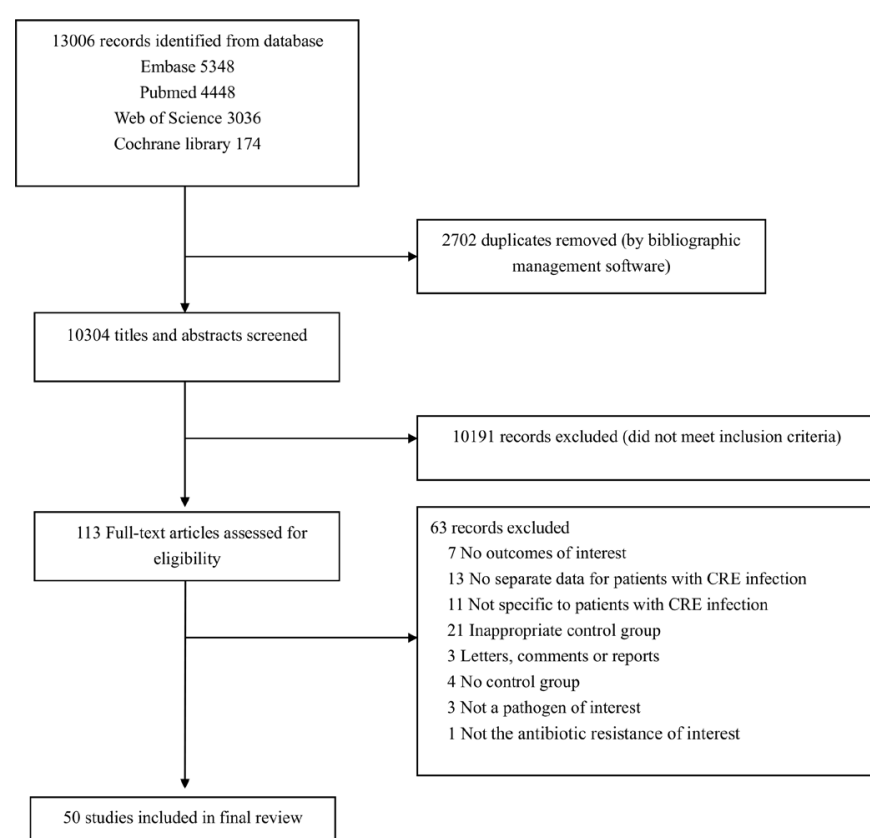

Figure 1 Flowchart of the study selection process for the meta-analysis. CRE, carbapenem-resistant Enterobacteriaceae.

of the studies were conducted in Asia $(n=24)$, followed by the Americas $(n=15)$ and Europe $(n=9)$, with only one study conducted in Africa. We also included a multiregion study that contained data from Asia, Africa and South America ${ }^{79}$ Most of the studies were conducted in high-income countries $(\mathrm{n}=27)$ and upper middle-income countries $(n=19)$, only three studies were conducted in lower middle-income countries and no study conducted in a low-income country met the criteria. Most studies $(n=39)$ reported mortality outcomes of infections that were due to Klebsiella pneumoniae pathogens, while two studies reported mortality outcomes of infections that were due to E. coli, and nine studies reported mortality outcomes regardless of the specific species of Enterobacteriaceae. Nearly half of the studies $(n=24)$ evaluated infected patients regardless of specific infection type. Among the studies that focused on specific sites of infection, bloodstream infection was the most frequent type $(\mathrm{n}=21)$, followed by urinary tract infection $(\mathrm{n}=3)$, and one study each for neurosurgical infection and pneumonia. Among the 50 studies included, most were cohort studies $(n=29)$. In the other 21 case-control studies, the mortality outcomes were measured using a cohort study design, so these studies were assessed as cohort studies in our quality appraisal. The NOS assessment for the risk of bias of all included studies was summarised in online supplemental appendix 4. According to the NOS scores, 46 were categorised as having a low risk of bias (scoring 7 to 9) and only four studies were categorised as having the moderate risk of bias (scoring 4to 6).

\section{Meta-analysis results}

Among the 50 studies included, 10 different measures of mortality were reported. In-hospital mortality $(n=31)$ was most frequently reported, followed by 28-day mortality $(\mathrm{n}=9)$, 30-day mortality $(\mathrm{n}=8)$, mortality attributable to infection $(n=8)$, 14-day mortality $(n=4)$ and mortality in ICU $(n=4)$. The mortality rates that were not commonly reported were 7 -day mortality $(n=2), 6$-day mortality $(\mathrm{n}=1)$, 21-day mortality $(\mathrm{n}=1)$ and 30 -day mortality in the ICU $(n=1)$. The meta-analysis result for the overall mortality based on the measure of RR (RR, 2.14, 95\% CI 1.85 to $2.48 ; \mathrm{I}^{2}=80.0 \%$ ) (figure 2) and $\mathrm{RD}$ (RD, 0.22, $95 \%$ CI 0.18 to $0.26 ; \mathrm{I}^{2}=78.0 \%$ ) (figure 3 ) suggested that carbapenem resistance was associated with increased risk of overall mortality, although a high level of heterogeneity was detected in these results.

The results of meta-analyses for different mortality outcome types showed that the $\mathrm{I}^{2}$ for the pooled RR and $\mathrm{RD}$ was 0 in the studies that reported 14-day mortality, 6-day or 7-day mortality and mortality in ICU, demonstrating low heterogeneity (table 2). Among these three groups, the lowest pooled RR (1.17, 95\% CI 1.08 to 1.28$)$ and RD $(0.09,95 \%$ CI 0.04 to 0.14$)$ was from the studies that reported mortality in the ICU. Although the pooled RR for 6-day or 7-day mortality (RR, 3.68, 95\% CI 2.32 to 5.83) was higher than that for 14-day mortality (RR, 1.70, $95 \%$ CI 1.24 to 2.35 ), the pooled RD for both groups was 0.18 . However, substantial heterogeneity was detected in the groups of studies that reported in-hospital mortality, 28-day or 30-day mortality, or mortality that was attributable to infection, which suggests other sources of heterogeneity.

\section{Stratified analysis}

To explore the source of heterogeneity between studies, we conducted a stratified analysis for each type of mortality outcome that had substantial heterogeneity. The potential sources of heterogeneity we explored were pathogens, geographical region, economic status of the country, source of infection, resistance mechanism type, sample size and publication year. One study ${ }^{79}$ was not included in our subgroup analysis by geographical region and country income level, because it was conducted in 10 countries with different economic status from three continents.

For in-hospital mortality, carbapenem resistance had a significant positive effect on the risk of death for patients infected with Enterobacteriaceae in most subgroups. However, in-hospital mortality was not significantly different in either relative or absolute terms between CRE infection and CSE infection in studies that focused on patients infected with E. coli pathogens (RR, 3.83, 95\% CI 0.46 to $31.78, p=0.214$; RD, $0.27,95 \%$ CI -0.06 to 0.59 , $\mathrm{p}=0.115)$ or oxacillinase(OXA)-producing Enterobacteriaceae $(\mathrm{RR}, 3.15,95 \%$ CI 0.45 to $21.96, \mathrm{p}=0.247$; RD, 0.24 , $95 \% \mathrm{CI}-0.05$ to $0.53, \mathrm{p}=0.110)$. In addition, no significant difference in pooled RR for in-hospital mortality was observed in studies that focused on patients with urinary tract infections (RR, 2.40, 95\% CI 0.82 to $7.03, \mathrm{p}=0.110$ ). The statistical test based on RR and RD showed that the effect of carbapenem resistance on mortality was not 
Table 1 Characteristics of included studies

\begin{tabular}{|c|c|c|c|c|c|}
\hline First author (year) & Study period & Country & Infection type & Pathogen & Mortality outcomes \\
\hline Alicino $(2015)^{30}$ & 2007.1-2014.12 & Italy & BSI & K.pneumoniae & 30-day mortality \\
\hline Balkhair $(2019)^{31}$ & 2007.1-2016.12 & Oman & $\mathrm{BSI}$ & K.pneumoniae & 30-day mortality \\
\hline Brizendine $(2015)^{33}$ & 2006-2012 & USA & UTI & K.pneumoniae & In-hospital mortality \\
\hline Chang $(2019)^{34}$ & $2014.1-2018.7$ & China & BSI & K.pneumoniae & $\begin{array}{l}\text { 7-day mortality, 28-day mortality, } \\
\text { In-hospital mortality }\end{array}$ \\
\hline Chang $(2011)^{35}$ & 2006.1-2008.12 & China & BSI & E.coli & $\begin{array}{l}\text { 14-day mortality, 28-day mortality, } \\
\text { In-hospital mortality }\end{array}$ \\
\hline Chiotos $(2018)^{36}$ & $2011.1-2016.7$ & USA & Mixed & Enterobacteriaceae & 30-day mortality \\
\hline $\begin{array}{l}\text { Cienfuegos-Gallet } \\
(2019)^{37}\end{array}$ & $\begin{array}{l}2014.2-3 ; 2014.10- \\
2015.9\end{array}$ & Colombia & Mixed & K.pneumoniae & 30-day mortality \\
\hline Fraenkel-Wandel (2016) ${ }^{41}$ & $2006-2012$ & Israel & BSI & K.pneumoniae & In-hospital mortality \\
\hline Gallagher $(2014)^{42}$ & 2005.6-2010.10 & USA & $\mathrm{BSI}$ & K.pneumoniae & In-hospital mortality \\
\hline Garbati $(2016)^{43}$ & 2012.3-2013.12 & Saudi Arabia & Mixed & Enterobacteriaceae & In-hospital mortality \\
\hline Gomez Rueda (2014) ${ }^{44}$ & 2008.1-2011.1 & Colombia & Mixed & K.pneumoniae & In-hospital mortality \\
\hline Hoxha $(2016)^{45}$ & 2012.11-2013.7 & Italy & Mixed & K.pneumoniae & 6-day mortality, 30-day mortality \\
\hline Huang $(2018)^{46}$ & 2017.1-2017.12 & China & Mixed & K.pneumoniae & In-hospital mortality \\
\hline Hussein $(2013)^{47}$ & 2006.1-2008.12 & Israel & BSI & K.pneumoniae & 30-day mortality \\
\hline Kotb $(2020)^{48}$ & 2011-2017 & Egypt & Mixed & Enterobacteriaceae & Mortality in ICU \\
\hline Lee $(2016)^{49}$ & 2013.1-2014.2 & Korea & Mixed & Enterobacteriaceae & $\begin{array}{l}\text { 28-day mortality, In-hospital } \\
\text { mortality }\end{array}$ \\
\hline Mouloudi (2010) ${ }^{55}$ & 2007.1-2008.12 & Greece & BSI & K.pneumoniae & $\begin{array}{l}\text { In-hospital mortality, mortality } \\
\text { in ICU, mortality attributable to } \\
\text { infection }\end{array}$ \\
\hline Ny $(2015)^{56}$ & 2011.1-2013.12 & USA & Mixed & K.pneumoniae & In-hospital mortality \\
\hline Orsi $(2013)^{57}$ & $2008.7-2011.6$ & Italy & Mixed & K.pneumoniae & In-hospital mortality \\
\hline Pan $(2019)^{58}$ & 2014 & China & Mixed & K.pneumoniae & In-hospital mortality \\
\hline Patel $(2008)^{59}$ & $2004.7-2006.6$ & USA & Mixed & K.pneumoniae & $\begin{array}{l}\text { In-hospital mortality, mortality } \\
\text { attributable to infection }\end{array}$ \\
\hline Pereira $(2015)^{60}$ & $2010.1-2013.1$ & USA & Mixed & K.pneumoniae & In-hospital mortality \\
\hline Pouch $(2015)^{61}$ & 2007.1-2010.12 & USA & UTI & Enterobacteriaceae & In-hospital mortality \\
\hline Qureshi $(2012)^{62}$ & 2011.1-2014.12 & USA & BSI & K.pneumoniae & 28-day mortality \\
\hline Sánchez-Romero (2011) ${ }^{63}$ & 2009.1-2009.12 & Spain & Mixed & K.pneumoniae & 14-day mortality \\
\hline Schwaber $(2008)^{64}$ & 2003.9-2006.12 & Israel & Mixed & K.pneumoniae & In-hospital mortality \\
\hline Shilo $(2013)^{65}$ & 2006.1-2009.12 & Israel & UTI & K.pneumoniae & In-hospital mortality \\
\hline Simkins $(2014)^{66}$ & 2006.1-2010.12 & USA & Mixed & K.pneumoniae & In-hospital mortality \\
\hline $\operatorname{Tian}(2016)^{67}$ & 2011.1-2015.12 & China & BSI & K.pneumoniae & $\begin{array}{l}\text { In-hospital mortality, mortality } \\
\text { attributable to infection, 28-day } \\
\text { mortality }\end{array}$ \\
\hline Torres-Gonzalez (2016) ${ }^{68}$ & 2013.11-2015.7 & Mexico & Mixed & Enterobacteriaceae & Mortality attributable to infection \\
\hline
\end{tabular}

Continued 


\begin{tabular}{|c|c|c|c|c|c|}
\hline First author (year) & Study period & Country & Infection type & Pathogen & Mortality outcomes \\
\hline Trecarich (2016)i69 & 2010.1-2014.6 & Italy & BSI & K.pneumoniae & 21-day mortality \\
\hline Vardakas $(2015)^{71}$ & $2006.1-2009.10$ & Greece & Mixed & K.pneumoniae & Mortality in ICU \\
\hline Wang $(2018)^{72}$ & 2010.1-2014.12 & China & Mixed & K.pneumoniae & In-hospital mortality \\
\hline Zhang $(2018)^{74}$ & 2011.1-2014.12 & China & BSI & K.pneumoniae & $\begin{array}{l}\text { 7-day mortality, } 28 \text {-day mortality, } \\
\text { In-hospital mortality }\end{array}$ \\
\hline Zheng $(2018)^{75}$ & 2014.1-2016.12 & China & BSI & K.pneumoniae & 28-day mortality \\
\hline Zheng $(2020)^{76}$ & 2012-2017 & China & $\begin{array}{l}\text { Neurosurgical } \\
\text { infection }\end{array}$ & Enterobacteriaceae & Mortality attributable to infection \\
\hline Stewardson $(2019)^{79}$ & $2014.8-2015.6$ & 10 countries & BSI & Enterobacteriaceae & In-hospital mortality \\
\hline
\end{tabular}

BSI, bloodstream infection; E. coli, Escherichia; K.pneumoniae, Klebsiella pneumoniae; UTI, urinary tract infection.

significantly different between the subgroups (online supplemental table 2).

For 28-day or 30-day mortality, the subgroup analysis showed no significant difference in the mortality for CRE and CSE infections that were due to mixed
Enterobacteriaceae pathogens (RR, 1.78, 95\% CI 0.57 to $5.60, \mathrm{p}=0.321 ; \mathrm{RD}, 0.05,95 \% \mathrm{CI}-0.03$ to $0.13, \mathrm{p}=0.213)$. The results of the statistical tests based on RR showed that the later studies, those that were published from 2017 to 2020, reported higher RR for 28-day or 30-day mortality

\begin{tabular}{|c|c|c|c|c|}
\hline author & year measurement & & $\operatorname{RR}(95 \% \mathrm{Cl})$ & $\begin{array}{l}\% \\
\text { Weight }\end{array}$ \\
\hline Patel & 2008 inhospital mortality & & $2.40(1.54,3.73)$ & 2.43 \\
\hline Schwaber & 2008 inhospital mortality & & $3.50(1.63,7.51)$ & 1.68 \\
\hline Daikos & $200914 d$ mortality & & $2.54(1.26,5.12)$ & 1.81 \\
\hline Mouloudi & 2010 inhospital mortality & & $1.65(0.95,2.86)$ & 2.16 \\
\hline Chang & 2011 inhospital mortality & & $1.88(1.32,2.69)$ & 2.64 \\
\hline Sánchez-Romero 2 & $201114 \mathrm{~d}$ mortality & & $1.50(0.86,2.63)$ & 2.14 \\
\hline Ben-David & 2012 inhospital mortality & & $2.93(1.90,4.53)$ & 2.45 \\
\hline Liu & 2012 inhospital mortality & & $1.50(0.94,2.39)$ & 2.37 \\
\hline Qureshi & $201228 \mathrm{~d}$ mortality & & $1.73(0.90,3.31)$ & 1.92 \\
\hline Correa & 2013 inhospital mortality & & $1.82(0.93,3.54)$ & 1.88 \\
\hline HUSAsein & 2013 30d mortality & & $1.51(1.11,2.04)$ & 2.76 \\
\hline Orsi & 2013 inhospital mortality & & $1.39(0.74,2.62)$ & 1.97 \\
\hline Shilo & 2013 inhospital mortality & & $1.15(0.77,1.71)$ & 2.53 \\
\hline Gallagher & 2014 inhospital mortality & & $1.36(0.89,2.09)$ & 2.46 \\
\hline Gomez Rueda & 2014 inhospital mortality & & $1.55(1.00,2.40)$ & 2.45 \\
\hline Mclaughlin & 2014 inhospital mortality & & $2.86(1.05,7.75)$ & 1.26 \\
\hline Simkins & 2014 inhospital mortality & & $6.00(1.74,20.64)$ & 0.95 \\
\hline Alicino & 2015 30d mortality & & $1.54(1.13,2.10)$ & 2.74 \\
\hline Brizendine & 2015 inhospital mortality & & $11.64(1.37,98.62$ & 0.40 \\
\hline Cubero & 2015 inhospital mortality & & $3.15(0.45,21.96)$ & 0.47 \\
\hline Ny & 2015 inhospital mortality & & $1.40(0.48,4.10)$ & 1.15 \\
\hline Pereira & 2015 inhospital mortality & & $1.62(0.79,3.31)$ & 1.78 \\
\hline Pouch & 2015 inhospital mortality & & $3.00(1.17,7.67)$ & 1.35 \\
\hline Ulu & 2015 mortality in ICU & & $0.88(0.58,1.33)$ & 2.49 \\
\hline Vardakas & 2015 mortality in ICU & & $1.24(0.86,1.79)$ & 2.62 \\
\hline Fraenkel-Wandel 2 & 2016 inhospital mortality & & $1.63(1.24,2.14)$ & 2.83 \\
\hline Garbati & 2016 inhospital mortality & & $2.57(1.07,6.21)$ & 1.45 \\
\hline Hoxha & 2016 30d mortality & & $3.00(1.65,5.45)$ & 2.05 \\
\hline Lee & 2016 inhospital mortality & & $1.00(0.27,3.70)$ & 0.88 \\
\hline Tian & 2016 inhospital mortality & & $2.15(1.19,3.88)$ & 2.06 \\
\hline Torres-Gonzalez 2 & 2016 mortality attributable to infection & & $1.50(0.43,5.28)$ & 0.93 \\
\hline Trecarichi & $201621 \mathrm{~d}$ mortality & & $3.59(2.26,5.71)$ & 2.38 \\
\hline Villegas & 2016 inhospital mortality & & $2.12(1.59,2.84)$ & 2.79 \\
\hline Meng & 2017 inhospital mortality & & $11.76(1.46,94.93$ & 0.42 \\
\hline Chiotos & $201830 \mathrm{~d}$ mortality & & $4.65(0.68,31.72)$ & 0.48 \\
\hline Huang & 2018 inhospital mortality & & $2.59(1.80,3.72)$ & 2.62 \\
\hline Wang & 2018 inhospital mortality & & $11.50(2.87,46.10$ & ) 0.81 \\
\hline Xiao & 2018 30d mortality & & $3.81(2.81,5.16)$ & 2.76 \\
\hline Zhang & 2018 inhospital mortality & & $2.22(0.90,5.48)$ & 1.41 \\
\hline Zheng & 2018 28d mortality & & $2.77(1.95,3.94)$ & 2.65 \\
\hline Balkhair & $201930 \mathrm{~d}$ mortality & & $2.63(2.01,3.43)$ & 2.84 \\
\hline Chang & 2019 inhospital mortality & & $3.79(2.59,5.56)$ & 2.58 \\
\hline Cienfuegos-Gallet 2 & $201930 \mathrm{~d}$ mortality & & $2.05(1.27,3.32)$ & 2.33 \\
\hline $\mathrm{Li}$ & 201930 d mortality in ICU & & $2.60(1.75,3.87)$ & 2.54 \\
\hline Liu & 2019 30d mortality & & $3.45(1.76,6.75)$ & 1.87 \\
\hline Pan & 2019 inhospital mortality & & $3.17(2.09,4.80)$ & 2.49 \\
\hline Stewardson & 2019 inhospital mortality & & $1.74(1.19,2.55)$ & 2.58 \\
\hline Kotb & 2020 mortality in ICU & & $1.18(1.08,1.29)$ & 3.11 \\
\hline Zheng & 2020 mortality attribute to infection & & $5.70(3.22,10.08)$ & 2.11 \\
\hline Zuo & 2020 inhospital mortality & & $1.73(1.00,3.00)$ & 2.17 \\
\hline Overall (I-squared & $d=80.0 \%, p=0.000)$ & $\diamond$ & $2.14(1.85,2.47)$ & 100.00 \\
\hline \multicolumn{5}{|c|}{ NOTE: Weights are from random effects analysis } \\
\hline & & & & \\
\hline
\end{tabular}

Figure 2 Forest plot of overall mortality in patients with carbapenem-resistant Enterobacteriaceae (CRE) versus carbapenemsusceptible Enterobacteriaceae (CSE) infections (outcome measure=relative risk). ICU, intensive care unit. 


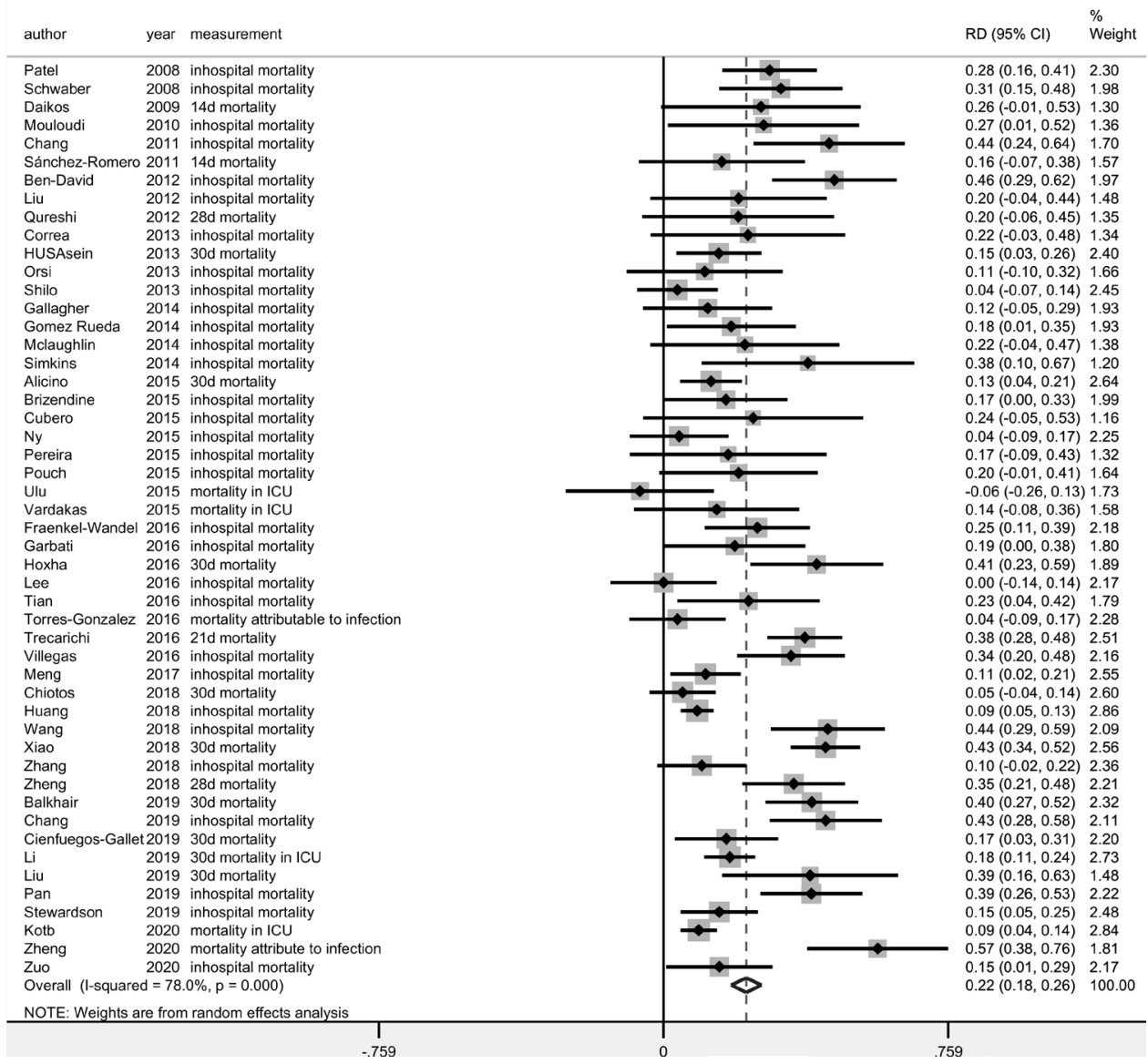

Figure 3 Forest plot of overall mortality in patients with carbapenem-resistant Enterobacteriaceae (CRE) versus carbapenemsusceptible Enterobacteriaceae (CSE) infections (outcome measure=risk difference). ICU, intensive care unit.

for patients who were infected with CRE versus CSE $(p=0.006)$ than did studies that were published earlier. The statistical test results for 28-day or 30-day mortality showed that the pooled RD in studies with fewer than 100 patients was higher than that in studies with 100-200 patients. Although the pooled RD in studies with more than 200 patients was highest, the heterogeneity in this group was high and should be interpreted with caution (online supplemental table 3).

For mortality attributable to infection, the one study conducted in Europe with a sample size of fewer than 100 has found no significant difference in the risk of death for CRE and CSE infection (RR, 1.98, 95\% CI 0.61 to 6.43, $\mathrm{p}=0.255$; RD, $0.13,95 \% \mathrm{CI},-0.07$ to $0.34, \mathrm{p}=0.195$ ), nor the study that focused on patients infected with OXAproducing Enterobacteriaceae (RR, 1.50, 95\% CI 0.43 to $5.28, \mathrm{p}=0.528$; RD, $0.04,95 \% \mathrm{CI},-0.09$ to $0.17, \mathrm{p}=0.572$ ). The results of statistical tests based on RD indicated that the effect of carbapenem resistance on attributable mortality was varied by the type of infection $(\mathrm{p}=0.075)$. Patients with neurosurgical infection were at greater risk of attributable death that was due to CRE infection than other types of infection (online supplemental table 4).

\section{Meta-regression}

To further explore whether the effect of carbapenem resistance on mortality differs by the variables of pathogens, geographical region, economic status of the country, source of infection, resistance mechanism type, sample size and publication year, we conducted the univariate meta-regression in the groups of mortality outcome type with more than 10 studies. The meta-regression results based on RD showed that the effect of carbapenem resistance on mortality was not influenced significantly by all the variables (online supplemental table 5, 6). However, in terms of $\mathrm{RR}$, the meta-regression for in-hospital mortality suggested that the influence of carbapenem resistance on in-hospital mortality in studies published between 2017 and 2020 was significantly greater than that in studies published between 2011 and 2013 (coefficient $=-0.447, \mathrm{p}=0.027$ ) and in studies published from 2014 to 2016 (coefficient=-0.343, $\mathrm{p}=0.061$ ) (online supplemental table 7). The results of the meta-regression for 28-day or 30-day mortality based on RR were similar to the results for in-hospital mortality. Moreover, the effect of carbapenem resistance on mortality at 28-day or 30-day tends to increase with the year of publication (coefficient $=-0.0001, \mathrm{p}=0.006$ ) (online supplemental table 8)

\section{Sensitivity analysis}

To assess the influence of individual studies on the results, we performed a sensitivity analysis by removing one study at a time and recalculated the pooled RRs of the overall mortality among the remaining studies using 


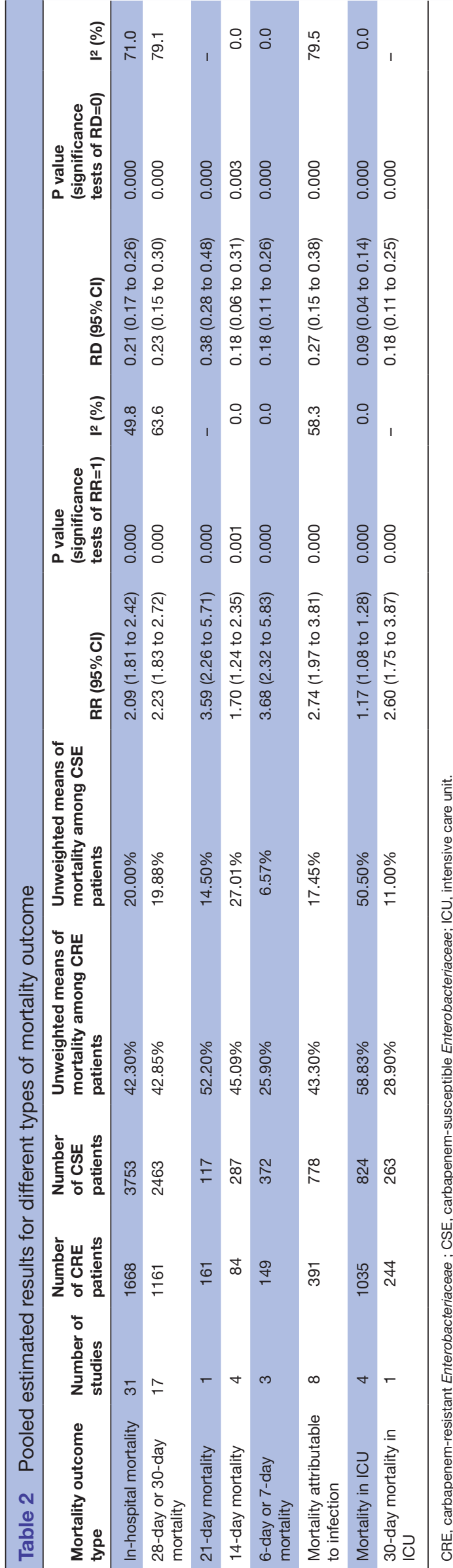

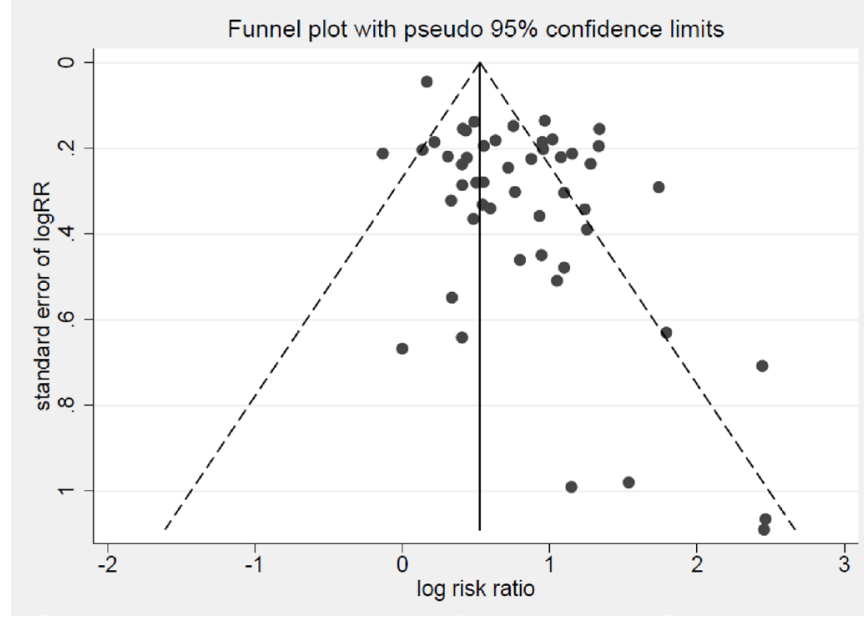

Figure 4 Funnel plot of studies evaluating mortality of patients with infections due to carbapenem-resistant compared with carbapenem-susceptible Enterobacteriaceae.

random effects meta-analysis. We found that the direction of the effect did not change when any one study was excluded, which indicates the stability of the results of the meta-analysis.

\section{Publication bias}

Publication bias was assessed by a funnel plot (figure 4). Slight asymmetricity was observed in the funnel plots and the points were heavily distributed at the top right, implying a lack of smaller studies that showed a negative association between carbapenem resistance and mortality.

\section{DISCUSSION}

This study systematically reviewed 50 studies and provides a comprehensive analysis of the impact of carbapenem resistance on mortality among patients infected with Enterobacteriaceae. Our analysis suggests that, for any type of mortality outcome, carbapenem resistance was associated with a greater probability of death for patients infected with CRE than that for patients infected with CSE. The results are consistent with the direction of previous meta-analyses of the association between carbapenem resistance and mortality among patients infected with Enterobacteriaceae. ${ }^{13} 14$

As for the risk factors for worse mortality outcomes in patients with CRE infections, previous studies usually explained higher mortality among patients with CRE infection as being due to patient-related, infection-related, treatment-related and organism-related factors. ${ }^{13} 141680$ Twenty studies included in this review conducted multivariate analyses to identify the risk factors for mortality among patients infected with Enterobacteriaceae. After controlling for patient-related factors like age, sex, the severity of underlying illness and comorbidities, three studies $^{475167}$ found that carbapenem resistance was not associated with increased mortality risk; however, 14 studies found that carbapenem resistance remained an independent predictor of mortality. Previous studies 
also considered therapeutic interventions as important risk factors for increased mortality in CRE infection, as administration of initial antibiotic therapy with in-vitro activity is more likely to be delayed in patients with CRE infection. ${ }^{32} 334059626774$ Several studies included in this research have suggested that the effect of carbapenem resistance was probably mediated by inappropriate initial therapy. ${ }^{37} 4051$ This finding was supported by a recent review of 11 studies that used a meta-regression analysis to identify a significant association between the proportion of patients who received appropriate initial antibiotic therapy and mortality. ${ }^{16}$ However, nine studies included in our review 323841476267717374 did not identify an association between early appropriate antibiotic therapy and mortality after adjustment for some confounding factors. Instead, some studies found that other treatment methods were important risk factors of mortality. For example, a recent meta-analysis including seven studies showed that monotherapy treatment was associated with significantly higher mortality than combination therapy for patients with CRE infections. ${ }^{14}$ In addition, some studies ${ }^{72} 73$ have suggested that other therapies, such as adjunctive therapy, tigecycline therapy and the use of aminoglycoside, may be associated with mortality among patients infected with Klebsiella pneumoniae. The increased mortality among patients with CRE infections might also be related to the increased virulence of carbapenemaseproducing strains. Two studies included in this metaanalysis showed that isolation of the Klebsiella pneumoniae carbapenemase(KPC)-positive strain was a predictor of mortality among patients infected with Klebsiella pneumoniae independent of the appropriateness of initial treatment and patient characteristics, ${ }^{41}{ }_{55}$ while another study ${ }^{47}$ found that Klebsiella pneumoniae carbapenemase(KPC)positive status was not associated with mortality when the virulence score was included in the multivariate analysis. As most of the included studies we reviewed did not provide the mortality outcomes after adjusting for confounding factors, the pooled-adjusted effect measures was not caculated.

To investigate the heterogeneity across the studies, we performed stratified analysis and meta-regression based on the type of mortality outcome. In terms of RR, the meta-regression analysis for in-hospital mortality showed that the effect of carbapenem resistance on in-hospital mortality was greater in studies published in 2017-2020 than it was in studies published in 2011-2013 and 20142016. The statistical test and meta-regression analyses for 28-day and 30-day mortality showed similar results. The increasing effect of carbapenem resistance on mortality with the publication year could be explained by the increasingly limited availability of effective antibiotics and the development of CRE against some key antibiotics, such as colistin, ${ }^{81}$ resulting in increasing difficulty in treating CRE infection. As one study showed, ${ }^{16}$ the proportion of patients with carbapenem-resistant Klebsiella pneumoniae who received appropriate initial antibiotic therapy did not change over time. In contrast, mortality from CSE infection has tended to decrease in recent years, and the unweighted mean of in-hospital mortality and 28-day and 30-day mortality among CSE patients in studies conducted from 2017 to 2020 is $11.69 \%$ and $13.43 \%$, respectively, the lowest of the studies we reviewed. This change could be due to the development of medical technology and medical treatment, which may enlarge the relative differences in mortality between CRE and CSE infections. In addition, the statistical test for mortality attributable to infection identified a significant difference between infection types, as carbapenem resistance in patients with neurosurgical infection had a significantly greater effect on mortality compared with other types of infection, perhaps because of difficulty in treating CRE meningitis/encephalitis in neurosurgery. ${ }^{74}$ In terms of RR, the statistical test showed that, compared with studies with fewer than 100 patients, carbapenem resistance had a greater effect on 28-day and 30-day mortality in studies with 100-200 patients, indicating that the absolute RD of mortality between CRE and CSE infection tends to be more stable with larger sample size.

To our knowledge, this study offers the most comprehensive meta-analysis so far of the impact of carbapenem resistance on mortality among patients infected with Enterobacteriaceae. Nearly, 20 new studies published in the last 3 years have been included in our study. In addition, the meta-analysis was conducted in different groups of mortality outcomes, which may help address the potential heterogeneity caused by mortality measurements. Moreover, this review is the first to explore the source of heterogeneity among studies using statistical tests and meta-regression analyses of variables related to countries' economic status and geographical region. Moreover, this is the first review to explore the source of heterogeneity across studies using statistical tests and meta-regression analysis of potential variables and to consider the country's economic status and geographical region in assessing the association between carbapenem resistance and mortality among patients infected with Enterobacteriaceae.

Our study also has several limitations. First, among studies focusing on specific pathogens, we only included studies that focused on two clinically important Enterobacteriaceae species, Klebsiella pneumoniae and Escherichia coli. Second, we only included studies published in English. Third, we only calculated the unadjusted results, so many confounding factors, such as patients' health conditions and therapy options, were not adjusted in the analysis because of data limitations. In addition, we were unable to conduct the stratified analysis and meta-regression for all kinds of mortality measurements because of insufficient data. Finally, the comparison in our research is currently limited to high-income and upper middle-income countries from the Americas, Asia and Europe due to insufficient data.

Our findings reinforced previous results regarding the positive effect of carbapenem resistance on mortality for patients infected with Enterobacteriaceae. These findings implied that patients with CRE infection still face a 
greater risk of death compared with patients with CSE infection. Furthermore, this study has identified an increasing effect of carbapenem resistance on mortality over time, especially for 28-day to 30-day mortality, which may reflect the difficulty of the CRE infection treatment in clinical practice and emphasise the urgent need to develop new antibiotics and appropriate treatment to reduce the death risk. Our results also suggested that patients with neurosurgical infection were at greater risk of attributable death that was due to CRE infection than other types of infection. Thus, more attention should be paid to CRE infection in patients with neurosurgery in clinical practice. In addition, no significant differences in the effect of carbapenem resistance on mortality for different geographical regions and economic status were observed in our study, which may result from the limited data. More studies from different countries, especially low-income countries, are needed to provide comprehensive data for further analysis stratified by geographical region and economic status.

\section{CONCLUSIONS}

Our results indicate that patients with CRE infection still face a greater risk of death than patients with CSE infection do, and an urgent need to develop new antibiotics and appropriate treatment to reduce the death risk. Future studies should address additional countries to provide comprehensive data and sound evidence from which to draw resources to fight CRE-related mortality and suggest the way forward to alleviate its implications.

\section{Author affiliations}

${ }^{1}$ Department of Economics and Trade, College of Economics and Management, China Agricultural University, Beijing, China

${ }^{2}$ Department of Health Policy and Behavioral Sciences, School of Public Health, Georgia State University, Atlanta, GA, USA

${ }^{3}$ Department of Economics, School of Economics, Zhejiang Gongshang University, Hangzhou, China

${ }^{4}$ Department of Basic Veterinary Medicine, College of Veterinary Medicine, China Agricultural University, Beijing, China

${ }^{5}$ CAS Key Laboratory of Pathogenic Microbiology and Immunology, Institute of Microbiology, Chinese Academy of Sciences, Beijing, China

${ }^{6}$ Agricultural Bio-pharmaceutical Laboratory, College of Chemistry and Pharmaceutical Sciences, Qingdao Agricultural University, Qingdao, China ${ }^{7}$ State Key Laboratory for Infectious Disease Prevention and Control, Collaborative Innovation Center for Diagnosis and Treatment of Infectious Disease, Chinese Center for Disease Control and Prevention, Beijing, Beijing, China

${ }^{8}$ Clinical Laboratory Medicine, The Second Affiliated Hospital of Zhejiang University, Zhejiang University, Hangzhou, China

${ }^{9}$ Beijing Key Laboratory of Detection Technology for Animal-Derived Food Safety, College of Veterinary Medicine, China Agricultural University, Beijing, China

${ }^{10}$ Department of Zoology, University of Oxford, Oxford, UK

Contributors All authors were involved in the design and development of the study. The review was designed by XF, RoZ, RuZ, JL, JS, TRW and YW. The literature search in electronic databases was conducted by JZ, SS, SC and XZ. RuZ and JZ screened all studies for inclusion into the systematic review and performed the assessments of risk bias for all studies with the assistance of XZ, SS, SC and XF. RuZ and JZ performed data extraction. RuZ and JZ conducted data analysis and interpretation with assistance of XZ, SS, SC and XF. RuZ drafted the manuscript and YS, ZL, JL, RoZ, JS, TRW, YW, XF revised it critically for important intellectual content. All authors contributed to drafting and revision of the article and have reviewed the results and approved the final version of the manuscript. The corresponding author (XF) is the guarantor and attests that all listed authors meet authorship criteria and that no others meeting the criteria have been omitted.

Funding This work was supported by the National Natural Science Foundation of China (NSFC) (Grant numbers 81861138051, 81861138052 and 81861138053 ).

Competing interests None declared.

Patient consent for publication Not applicable.

Ethics approval This study does not involve human participants or animal subjects.

Provenance and peer review Not commissioned; externally peer reviewed.

Data availability statement All data relevant to the study are included in the article or uploaded as supplementary information. Data sharing not applicable because all data relevant to the study are included in the article or uploaded as supplementary information.

Supplemental material This content has been supplied by the author(s). It has not been vetted by BMJ Publishing Group Limited (BMJ) and may not have been peer-reviewed. Any opinions or recommendations discussed are solely those of the author(s) and are not endorsed by BMJ. BMJ disclaims all liability and responsibility arising from any reliance placed on the content. Where the content includes any translated material, BMJ does not warrant the accuracy and reliability of the translations (including but not limited to local regulations, clinical guidelines, terminology, drug names and drug dosages), and is not responsible for any error and/or omissions arising from translation and adaptation or otherwise.

Open access This is an open access article distributed in accordance with the Creative Commons Attribution Non Commercial (CC BY-NC 4.0) license, which permits others to distribute, remix, adapt, build upon this work non-commercially, and license their derivative works on different terms, provided the original work is properly cited, appropriate credit is given, any changes made indicated, and the use is non-commercial. See: http://creativecommons.org/licenses/by-nc/4.0/.

ORCID iD

Ruyin Zhou http://orcid.org/0000-0002-5731-5746

\section{REFERENCES}

1 Center for Disease Control and Prevention(US). Clinicians: information about CRE, 2019. Available: https://www.cdc.gov/hai/ organisms/cre/cre-clinicians.html [Accessed 19 Dec 2020].

2 Birnbaum J, Kahan FM, Kropp H, et al. Carbapenems, a new class of beta-lactam antibiotics. discovery and development of imipenem/ cilastatin. Am J Med 1985;78:3-21.

3 Paterson DL, Ko W-C, Von Gottberg A, et al. Antibiotic therapy for Klebsiella pneumoniae bacteremia: implications of production of extended-spectrum beta-lactamases. Clin Infect Dis 2004;39:31-7.

4 Osano E, Arakawa Y, Wacharotayankun R, et al. Molecular characterization of an enterobacterial metallo beta-lactamase found in a clinical isolate of Serratia marcescens that shows imipenem resistance. Antimicrob Agents Chemother 1994;38:71-8.

5 Lauretti L, Riccio ML, Mazzariol A, et al. Cloning and characterization of blaVIM, a new integron-borne metallo-beta-lactamase gene from a Pseudomonas aeruginosa clinical isolate. Antimicrob Agents Chemother 1999;43:1584-90.

6 Yigit H, Queenan AM, Anderson GJ, et al. Novel carbapenemhydrolyzing beta-lactamase, KPC-1, from a carbapenem-resistant strain of Klebsiella pneumoniae. Antimicrob Agents Chemother 2001;45:1151-61.

7 lovleva A, Doi Y. Carbapenem-Resistant Enterobacteriaceae. Clin Lab Med 2017;37:303-15.

8 Logan LK, Weinstein RA. The epidemiology of carbapenem-resistant Enterobacteriaceae: the impact and evolution of a global menace. $J$ Infect Dis 2017;215:S28-36.

9 European Centre for Disease Control and Prevention. Antimicrobial resistance surveillance in Europe 2018, 2019. Available: https://www. ecdc.europa.eu/en/publications-data/surveillance-antimicrobialresistance-europe-2018 [Accessed 19 Dec 2020].

10 World Health Organization. Global priority list of antibiotic-resistant bacteria to guide research, discovery, and development of new antibiotics, 2017. Available: https://www.who.int/medicines/ publications/global-priority-list-antibiotic-resistant-bacteria/en/ [Accessed 19 Dec 2020].

11 Budhram DR, Mac S, Bielecki JM, et al. Health outcomes attributable to carbapenemase-producing Enterobacteriaceae infections: a 
systematic review and meta-analysis. Infect Control Hosp Epidemiol 2020;41:37-43.

12 Soontaros S, Leelakanok N. Association between carbapenemresistant Enterobacteriaceae and death: a systematic review and meta-analysis. Am J Infect Control 2019;47:1200-12.

13 Martin A, Fahrbach K, Zhao Q, et al. Association Between Carbapenem Resistance and Mortality Among Adult, Hospitalized Patients With Serious Infections Due to Enterobacteriaceae: Results of a Systematic Literature Review and Meta-analysis. Open Forum Infect Dis 2018;5: ofy150.

$14 \mathrm{Xu} \mathrm{L}$, Sun X, Ma X. Systematic review and meta-analysis of mortality of patients infected with carbapenem-resistant Klebsiella pneumoniae. Ann Clin Microbiol Antimicrob 2017;16:18.

15 Kohler PP, Volling C, Green K, et al. Carbapenem resistance, initial antibiotic therapy, and mortality in Klebsiella pneumoniae bacteremia: a systematic review and meta-analysis. Infect Control Hosp Epidemiol 2017;38:1319-28.

16 Falagas ME, Tansarli GS, Karageorgopoulos DE, et al. Deaths attributable to carbapenem-resistant Enterobacteriaceae infections. Emerg Infect Dis 2014;20:1170-5.

17 Laxminarayan R, Heymann DL. Challenges of drug resistance in the developing world. BMJ 2012;344:e1567.

18 Ramos-Castañeda JA, Ruano-Ravina A, Barbosa-Lorenzo R, et al. Mortality due to KPC carbapenemase-producing Klebsiella pneumoniae infections: systematic review and meta-analysis: mortality due to KPC Klebsiella pneumoniae infections. $J$ Infect 2018;76:438-48.

19 Higgins JPT, Green S. Cochrane handbook for systematic reviews of interventions. Version 5.1.0. [updated March 2011]. London: Cochrane Collaboration, 2011. Available: https:// handbook-5-1. cochrane.org/ [Accessed 19 Dec 2020].

20 Moher D, Liberati A, Tetzlaff J, et al. Preferred reporting items for systematic reviews and meta-analyses: the PRISMA statement. BMJ 2009;339:b2535.

21 Suay-García B, Pérez-Gracia MT. Present and future of carbapenemresistant Enterobacteriaceae (CRE) infections. Antibiotics 2019;8:122.

22 World Bank. World bank country and lending groups[Internet] Data, 2019. Available: https://datahelpdesk.worldbank.org/ knowledgebase/articles/906519-world-bank-country-and-lendinggroups [Accessed 20 Jun 2020].

23 Sinclair JC, Bracken MB. Clinically useful measures of effect in binary analyses of randomized trials. J Clin Epidemiol 1994;47:881-9.

24 Sackett DL, Deeks JJ, Altman DG. Down with odds ratios! BMJ Evid Based Med 1996;1:164-6.

25 Schmidt CO, Kohlmann T. When to use the odds ratio or the relative risk? Int J Public Health 2008;53:165-7.

26 Noordzij M, van Diepen M, Caskey FC, et al. Relative risk versus absolute risk: one cannot be interpreted without the other. Nephrol Dial Transplant 2017;32:ii13-18.

27 Schulz KF, Altman DG, Moher D, et al. Consort 2010 statement: updated guidelines for reporting parallel group randomised trials. Int J Surg 2011;9:672-7.

28 DerSimonian R, Laird N. Meta-analysis in clinical trials. Control Clin Trials 1986;7:177-88.

29 Wells GA, Shea B, O'Connell D. The Newcastle-Ottawa scale (NOS) for assessing the quality of nonrandomised studies in meta-analyses. Ottawa: Ottawa Hospital Research Institute, 2014.

30 Alicino C, Giacobbe DR, Orsi A, et al. Trends in the annual incidence of carbapenem-resistant Klebsiella pneumoniae bloodstream infections: a 8-year retrospective study in a large teaching hospital in northern Italy. BMC Infect Dis 2015;15:415

31 Balkhair A, Al-Muharrmi Z, Al'Adawi B, et al. Prevalence and 30-day all-cause mortality of carbapenem-and colistin-resistant bacteraemia caused by Acinetobacter baumannii, Pseudomonas aeruginosa, and Klebsiella pneumoniae: description of a decade-long trend. Int $J$ Infect Dis 2019;85:10-15.

32 Ben-David D, Kordevani R, Keller N, et al. Outcome of carbapenem resistant Klebsiella pneumoniae bloodstream infections. Clin Microbiol Infect 2012;18:54-60.

33 Brizendine KD, Richter SS, Cober ED, et al. Carbapenem-resistant Klebsiella pneumoniae urinary tract infection following solid organ transplantation. Antimicrob Agents Chemother 2015;59:553-7.

34 Chang $\mathrm{H}$, Wei J, Zhou W, et al. Risk factors and mortality for patients with bloodstream infections of Klebsiella pneumoniae during 2014-2018: clinical impact of carbapenem resistance in a large tertiary hospital of China. J Infect Public Health 2020;13:784-90.

35 Chang H-J, Hsu P-C, Yang C-C, et al. Risk factors and outcomes of carbapenem-nonsusceptible Escherichia coli bacteremia: a matched case-control study. J Microbiol Immunol Infect 2011;44:125-30.
36 Chiotos K, Tamma PD, Flett KB, et al. Increased 30-day mortality associated with carbapenem-resistant Enterobacteriaceae in children. Open Forum Infect Dis 2018;5:ofy222.

37 Cienfuegos-Gallet AV, Ocampo de Los Ríos AM, Sierra Viana P, et al. Risk factors and survival of patients infected with carbapenemresistant Klebsiella pneumoniae in a KPC endemic setting: a casecontrol and cohort study. BMC Infect Dis 2019;19:830.

38 Correa L, Martino MDV, Siqueira I, et al. A hospital-based matched case-control study to identify clinical outcome and risk factors associated with carbapenem-resistant Klebsiella pneumoniae infection. BMC Infect Dis 2013;13:80.

39 Cubero M, Cuervo G, Dominguez MÁ, et al. Carbapenem-resistant and carbapenem-susceptible isogenic isolates of Klebsiella pneumoniae ST101 causing infection in a tertiary hospital. BMC Microbiol 2015;15:177.

40 Daikos GL, Petrikkos P, Psichogiou M. Prospective observational study of the impact of VIM-1 metallo- $\beta$-lactamase on the outcome of patients with Klebsiella pneumoniae bloodstream infections. Antimicrob Agents Chemother 2009;53:1868-73.

41 Fraenkel-Wandel Y, Raveh-Brawer D, Wiener-Well Y, et al. Mortality due to blaKPC Klebsiella pneumoniae bacteraemia. J Antimicrob Chemother 2016;71:1083-7.

42 Gallagher JC, Kuriakose S, Haynes K, et al. Case-case-control study of patients with carbapenem-resistant and third-generationcephalosporin-resistant Klebsiella pneumoniae bloodstream infections. Antimicrob Agents Chemother 2014;58:5732-5.

43 Garbati MA, Sakkijha H, Abushaheen A. Infections due to carbapenem resistant Enterobacteriaceae among Saudi Arabian hospitalized patients: a matched case-control study. Biomed Res Int 2016;2016:1-9.

44 Rueda G V, Zuleta Tobon JJ. Risk factors for infection with carbapenem-resistant Klebsiella pneumoniae: a case-case-control study. Colomb Med 2014:45:54-60.

45 Hoxha A, Kärki T, Giambi C, et al. Attributable mortality of carbapenem-resistant Klebsiella pneumoniae infections in a prospective matched cohort study in Italy, 2012-2013. J Hosp Infect 2016;92:61-6.

46 Huang W, Qiao F, Zhang Y, et al. In-Hospital medical costs of infections caused by carbapenem-resistant Klebsiella pneumoniae. Clin Infect Dis 2018;67:S225-30.

47 Hussein K, Raz-Pasteur A, Finkelstein R, et al. Impact of carbapenem resistance on the outcome of patients' hospital-acquired bacteraemia caused by Klebsiella pneumoniae. $J$ Hosp Infect 2013:83:307-13

48 Kotb S, Lyman M, Ismail G, et al. Epidemiology of carbapenemresistant Enterobacteriaceae in Egyptian intensive care units using national healthcare-associated infections surveillance data, 20112017. Antimicrob Resist Infect Control 2020;9:2.

49 Lee HJ, Choi JK, Cho SY, et al. Carbapenem-Resistant Enterobacteriaceae: prevalence and risk factors in a single community-based hospital in Korea. Infect Chemother 2016:48:166-73.

$50 \mathrm{Li} \mathrm{Y,} \mathrm{Shen} \mathrm{H,} \mathrm{Zhu} \mathrm{C,} \mathrm{et} \mathrm{al.} \mathrm{Carbapenem-Resistant} \mathrm{Klebsiella}$ pneumoniae infections among ICU admission patients in central China: prevalence and prediction model. Biomed Res Int 2019;2019:1-10.

51 Liu J, Wang H, Huang Z, et al. Risk factors and outcomes for carbapenem-resistant Klebsiella pneumoniae bacteremia in oncohematological patients. J Infect Dev Ctries 2019;13:357-64.

52 Liu S-W, Chang H-J, Chia J-H, et al. Outcomes and characteristics of ertapenem-nonsusceptible Klebsiella pneumoniae bacteremia at a university hospital in Northern Taiwan: a matched case-control study. $J$ Microbiol Immunol Infect 2012;45:113-9.

53 McLaughlin MM, Advincula MR, Malczynski M, et al. Quantifying the clinical virulence of Klebsiella pneumoniae producing carbapenemase Klebsiella pneumoniae with a Galleria mellonella model and a pilot study to translate to patient outcomes. BMC Infect Dis 2014:14:31.

54 Meng X, Liu S, Duan J, et al. Risk factors and medical costs for healthcare-associated carbapenem-resistant Escherichia coli infection among hospitalized patients in a Chinese teaching hospital. BMC Infect Dis 2017;17:82.

55 Mouloudi E, Protonotariou E, Zagorianou A, et al. Bloodstream infections caused by metallo- $\beta$-lactamase/Klebsiella pneumoniae carbapenemase-producing $\mathrm{K}$. pneumoniae among intensive care unit patients in Greece: risk factors for infection and impact of type of resistance on outcomes. Infect Control Hosp Epidemiol 2010;31:1250-6.

56 Ny P, Nieberg P, Wong-Beringer A. Impact of carbapenem resistance on epidemiology and outcomes of nonbacteremic Klebsiella pneumoniae infections. Am J Infect Control 2015;43:1076-80. 
57 Orsi GB, Bencardino A, Vena A, et al. Patient risk factors for outer membrane permeability and KPC-producing carbapenem-resistant Klebsiella pneumoniae isolation: results of a double case-control study. Infection 2013;41:61-7.

58 Pan H, Lou Y, Zeng L, et al. Infections caused by carbapenemaseproducing Klebsiella pneumoniae: microbiological characteristics and risk factors. Microb Drug Resist 2019;25:287-96.

59 Patel G, Huprikar S, Factor SH, et al. Outcomes of carbapenemresistant Klebsiella pneumoniae infection and the impact of antimicrobial and adjunctive therapies. Infect Control Hosp Epidemiol 2008;29:1099-106.

60 Pereira MR, Scully BF, Pouch SM, et al. Risk factors and outcomes of carbapenem-resistant Klebsiella pneumoniae infections in liver transplant recipients. Liver Transpl 2015;21:1511-9.

61 Pouch SM, Kubin CJ, Satlin MJ, et al. Epidemiology and outcomes of carbapenem-resistant Klebsiella pneumoniae bacteriuria in kidney transplant recipients. Transpl Infect Dis 2015;17:800-9.

62 Qureshi ZA, Paterson DL, Peleg AY, et al. Clinical characteristics of bacteraemia caused by extended-spectrum $\beta$-lactamase-producing Enterobacteriaceae in the era of CTX-M-type and KPC-type $\beta$-lactamases. Clin Microbiol Infect 2012;18:887-93.

63 Sánchez-Romero I, Asensio A, Oteo J, et al. Nosocomial outbreak of VIM-1-producing Klebsiella pneumoniae isolates of multilocus sequence type 15: molecular basis, clinical risk factors, and outcome. Antimicrob Agents Chemother 2012;56:420-7.

64 Schwaber MJ, Klarfeld-Lidji S, Navon-Venezia S, et al. Predictors of carbapenem-resistant Klebsiella pneumoniae acquisition among hospitalized adults and effect of acquisition on mortality. Antimicrob Agents Chemother 2008;52:1028-33.

65 Shilo S, Assous MV, Lachish T, et al. Risk factors for bacteriuria with carbapenem-resistant Klebsiella pneumoniae and its impact on mortality: a case-control study. Infection 2013;41:503-9.

66 Simkins J, Muggia V, Cohen HW, et al. Carbapenem-resistant Klebsiella pneumoniae infections in kidney transplant recipients: a case-control study. Transpl Infect Dis 2014;16:775-82.

67 Tian L, Tan R, Chen Y, et al. Epidemiology of Klebsiella pneumoniae bloodstream infections in a teaching hospital: factors related to the carbapenem resistance and patient mortality. Antimicrob Resist Infect Control 2016;5:48.

68 Torres-González P, Ortiz-Brizuela E, Cervera-Hernandez ME, et al. Associated factors and outcomes for OXA-232 carbapenemresistant Enterobacteriaceae infections in a tertiary care centre in Mexico City: a case-control-control study. Diagn Microbiol Infect Dis 2016;86:243-8.

69 Trecarichi EM, Pagano L, Martino B, et al. Bloodstream infections caused by Klebsiella pneumoniae in onco-hematological patients: clinical impact of carbapenem resistance in a multicentre prospective survey. Am J Hematol 2016;91:1076-81.
70 Candevir Ulu A, Kurtaran B, Inal AS, et al. Risk factors of carbapenem-resistant Klebsiella pneumoniae infection: a serious threat in ICUs. Med Sci Monit 2015;21:219-24.

71 Vardakas KZ, Matthaiou DK, Falagas ME, et al. Characteristics, risk factors and outcomes of carbapenem-resistant Klebsiella pneumoniae infections in the intensive care unit. $J$ Infect 2015;70:592-9.

72 Wang Z, Qin R-R, Huang L, et al. Risk factors for carbapenemresistant Klebsiella pneumoniae infection and mortality of Klebsiella pneumoniae infection. Chin Med J 2018;131:56-62.

73 Xiao T, Yu W, Niu T, et al. A retrospective, comparative analysis of risk factors and outcomes in carbapenem-susceptible and carbapenemnonsusceptible Klebsiella pneumoniae bloodstream infections: tigecycline significantly increases the mortality. Infect Drug Resist 2018;11:595-606.

74 Zhang Y, Guo L-Y, Song W-Q, et al. Risk factors for carbapenemresistant K. pneumoniae bloodstream infection and predictors of mortality in Chinese paediatric patients. BMC Infect Dis 2018;18:248.

75 Zheng S-H, Cao S-J, Xu H, et al. Risk factors, outcomes and genotypes of carbapenem-nonsusceptible Klebsiella pneumoniae bloodstream infection: a three-year retrospective study in a large tertiary hospital in Northern China. Infect Dis 2018;50:443-51.

76 Guanghui Z, Jing L, Guojun Z, et al. Epidemiology and risk factors of neurosurgical bacterial meningitis/encephalitis induced by carbapenem resistant Enterobacteriaceae. J Infect Chemother 2020;26:101-6

77 Zuo Y, Zhao D, Song G, et al. Risk factors, molecular epidemiology, and outcomes of carbapenem-resistant Klebsiella pneumoniae infection for hospital-acquired pneumonia: a matched case-control study in eastern China during 2015-2017. Microb Drug Resist 2021;27:204-211.

78 Villegas MV, Pallares CJ, Escandón-Vargas K, et al. Characterization and clinical impact of bloodstream infection caused by carbapenemase-producing Enterobacteriaceae in seven Latin American countries. PLoS One 2016;11:e0154092.

79 Stewardson AJ, Marimuthu K, Sengupta S, et al. Effect of carbapenem resistance on outcomes of bloodstream infection caused by Enterobacteriaceae in low-income and middle-income countries (Panorama): a multinational prospective cohort study. Lancet Infect Dis 2019;19:601-10.

80 Paño Pardo JR, Serrano Villar S, Ramos Ramos JC, et al. Infections caused by carbapenemase-producing Enterobacteriaceae: risk factors, clinical features and prognosis. Enferm Infecc Microbiol Clin 2014;32:41-8.

81 Sader HS, Castanheira M, Duncan LR, et al. Antimicrobial susceptibility of Enterobacteriaceae and Pseudomonas aeruginosa isolates from United States medical centers stratified by infection type: results from the International network for optimal resistance monitoring (inform) surveillance program, 2015-2016. Diagn Microbiol Infect Dis 2018:92:69-74. 